\title{
Characterization of Intestinal Parasitosis in Pregant Women at Ram Janaki Hospital, Janakpurdham
}

\author{
Khushbu Yadav ${ }^{1}$, Satyam Prakash ${ }^{2}$, Basant Kumar Yadav ${ }^{3}$ \\ ${ }^{1}$ Lecturer, Department of Microbiology, Ram Janaki Technical Institute and Hospital, Janakpurdham, Nepal \\ ${ }^{2}$ Assistant Professor, Department of Biochemistry, Janaki Medical College, Janakpurdham, Nepal \\ ${ }^{3}$ Medical Officer, Department of Surgery, Janaki Medical College, Janakpurdham, Nepal
}

\begin{abstract}
Corresponding Author: Khushbu Yadav, Lecturer, Department of Microbiology, Ram Janaki Technical Institute and Hospital, Janakpurdham, Nepal, Email: meetkhushi20@gmail.com
\end{abstract}

\begin{abstract}
Objectives: The objective of this study was to determine the prevalence, detection and identification of intestinal parasites and its associated factors among pregnant women.

Methods: Total 264 stool samples were collected in a labeled dry, clean disinfectant free wide mouthed plastic container during antenatal visits at Ram Janaki Hospital, Janakpurdham and were examined by macroscopically and microscopically. The detection and identification of protozoal cysts, oocysts, trophozoites and helminthic eggs or larva was done by wet preparation and formalether sedimentation concentration technique. The data was analysed using SPSS 20 version and Microsoft Excel 2007. A Chi-square test was performed to predict the parasite detection using predictor variables. The $\mathrm{p}$-values $<0.05$ was considered as significant.
\end{abstract}

Results: The prevalence of intestinal parasitosis among pregnant women was $42 \%$. There was positive association of symptoms of intestinal parasitosis among pregnant women $(p<0.05)$. The most predominant intestinal parasites among study participants were E. histolytica (20\%) slightly dropped by G. lamblia (16\%) followed by Hook worm (13\%) and A. lumbricoides (11\%). The correlation between all the variables with intestinal parasites presence and absence was statistically significant $(p<0.05)$ but statistically insignificant for age and consumption of green leafy vegetables $(p>0.05)$.

Conclusion: The overall prevalence of intestinal parasitosis was relatively moderate. Lack of awareness, low hygienic and sanitation habits regarding parasitic infections were the major determinant factors for higher prevalence. Improving sanitation, awareness creation and public health programes should be organized at regular interval in community.

Keywords: Helminths, Hygiene, Infestation, Intestinal parasites, Pregnancy, Sanitation

\section{INTRODUCTION}

Pregnant women are one of the most vulnerable groups and often experience more severe infections due to their immune suppression during their pregnancy (Yakasai and Umar, 2013). Intestinal parasitic infections caused by protozoa and soil helminths which are transmitted faeco-orally through contaminated sources (Yadav and Prakash 2016; Rai et al. 2002). Most common intestinal parasites reported from Nepal are Ascaris lumbricoides, Hymenolepis nana, Hookworm, Trichuris trichiura, Giardia lamblia and Entamoeba histolytica. Of

Date of Submission: August 26, 2020

Published Online: December, 2020 the protozoal infections, amoebiasis and giardiasis are most frequently reported. Ascaris lumbricoides, Trichuris trichiura and Hookworms, collectively referred to as soil-transmitted helminths (STHs) which are the most common intestinal parasites (Yadav and Prakash 2016; Mehraj et al. 2006).

Globally, approximately, 4.5 billion people are at risk, more than 1 billion people become infected, and 450 million are ill from STHs (WHO, 2014). High prevalence of STHs is mainly related to poverty, poor living conditions, personal and environmental

Date of Acceptance: October 21, 2020

DOI: https:/ / doi.org/10.3126/tujm.v7i0.33851 
hygiene, sanitation, and water supply facilities (Ohaeri and Orji, 2013) low literacy rate, the habit of eating raw vegetables, walking barefoot, malnutrition and hot and humid tropical climate are some of the factors associated with the STH infections (Derso et al. 2016 ). Intestinal parasitic infections disturb pregnancy, directly or indirectly lead to a spectrum of adverse maternal and fetal/placental effects (Dotters-Katz et al. 2011). Infected pregnant women develop malnutrition; maternal anemia; total energy, protein, folate, and zinc loss (Stephenson et al. 2020); low pregnancy weight gain (Khor 2003) and increased vulnerability to other infections (Steketee, 2003). STH infections also show adverse outcomes on the offspring such as low birth weight, intrauterine fetal growth restriction, and perinatal mortality (Steketee 2003).

STHs is a significant community health problem, especially in developing countries of both Asia and Africa (De Silva et al. 2013). Soil transmitted helminthes infections are endemic in the communities where poor environmental sanitation and poor personal hygiene play an important role in transmission of STH infections. A. lumbricoides and Hookworms cause morbidity in humans in different ways by affecting nutritional equilibrium, inducing intestinal bleeding, inducing malabsorption of micronutrients, reducing growth, reducing food intake, causing complications such as obstruction rectal prolapsed, abscess and affecting congenital development (Mehraj et al. 2006).

Intestinal parasites (especially helminths) can be tissue dwelling or intestinal but all induce a dramatic expansion of the Th2 lymphocyte subset (Finkelman and Urban 2001). It remains unclear whether these Th2derived responses, including $\operatorname{IgE}$, eosinophilia, and mastocytosis are important in the protective immune response to the parasite, or are responsible for immunemediated pathology, or both but at least is a paraclinical marker of infection (Finkelman and Urban 2001).

To the best of our knowledge, institution-based information revealed that infection with protozoa and geohelminths is the primary disease among pregnant women (Hailu et al. 2020) but the prevalence and factors associated with parasitic infections are still unknown in Nepal. Therefore, this study was carried out to find out the prevalence of intestinal parasites among pregnant women attending for antenatal check ups at a tertiary care hospital and its association with various socio-demographic factors which will provide an opportunity to recommend the prevention, control and treatment of the intestinal parasites.

\section{MATERIAL AND METHODS}

\section{Study design and area}

This cross-sectional study was conducted among the pregnant women attending for antenatal care checkup at Department of Obstetrics and Gynecology at Ram Janaki Hospital, Janakpurdham. All the laboratory procedures were carried out at Microbiology Department of Clinical Pathology and Laboratory Medicine at Ram Janaki Hospital, Janakpurdham located in Dhanusha district at Province No. 2 of Nepal from June 2018 to September 2019. The random sampling technique was applied on 264 pregnant women.

\section{Data collection}

During the process of specimen collection from study participants, a structured questionnaire accompanying the queries about their sociodemographic variables (age, residence, occupation and religion), clinical history, hygienic practice and nutritional behavior were collected by face-to-face interviews. The data was collected by trained midwifery health professionals.

\section{Sample collection}

The stool samples were collected from the pregnant women. The containers were labeled with name, code number, date and time of collection. A labeled dry, clean disinfectant free wide mouthed plastic container was distributed to all study participants during antenatal visits to bring about 10 gms stool sample. They were advised not to contaminate the stool with water and urine. The collected stool samples were immediately preserved with $10 \%$ formalin solution.

\section{Inclusion and exclusion criteria}

Asymptomatic pregnant woman were included after informed consent. Women refusing to give consent and those who received prior treatment with anti-parasitic drugs before two weeks, severely ill or with previous diagnosis of infectious diseases as HIV/AIDS, HBV infection, syphilis, or toxoplasmosis were not enrolled in the study. Also, those participants who came with stool samples contaminated with water and urine were excluded from study.

\section{Laboratory investigation}

The collected stool samples were examined by macroscopic and microscopic examination. 


\section{Macroscopic examination}

The stool samples were observed for color, consistency, presence of blood and mucus, presence of adult worms and segments and other abnormalities.

\section{Microscopic examination}

The detection and identification of protozoal cysts, oocysts, trophozoites and helminthic eggs or larva by wet (normal saline and iodine) preparation and formalether sedimentation concentration technique (FECT) employed for all the stool specimens.

\section{Formal ether concentration techniques (FECT)}

About $0.5 \mathrm{gm}$ stool sample was transferred into 10 $\mathrm{ml}$ of normal saline in a glass container and mixed thoroughly. Two layers of gauze were placed in a funnel and strained the contents into a $15 \mathrm{ml}$ centrifuge tube. Then, $2.5 \mathrm{ml}$ of $10 \%$ formaldehyde and $1 \mathrm{ml}$ of ether were added to a test tube. The test tubes were mixed well and centrifuged at 1,000 rpm for 3 minutes. The supernatant was removed and sediment was further proceeded for wet mount preparation.

\section{Normal saline and iodine mount techniques}

The sediment was mixed well, prepared on two slides one with $2 \mathrm{ml}$ of normal saline and the other with $2 \mathrm{ml}$ of iodine solution, and covered with cover slide and detected under a microscope at 10X and 40X.

\section{Ethical consideration}

Informed verbal consent was obtained from the participants prior to the study before preceding the questionnaire and specimen collection. Work approval letter was taken from Ram Janaki Technical Institute and Ram Janaki Hospital, Janakpurdham, Nepal.

\section{Statistical analysis}

A descriptive analysis was done for the positivity among different age groups. The obtained data was analysed using SPSS 20 version and Microsoft Excel 2007. A Chi-square test was performed to predict the parasite detection using predictor variables for hand wash before eating, hand wash after defecation, green leafy vegetable consumption, use of latrine and footwear. The association of parasitic infection with sanitary practices and socio-demographic factors were also assessed by using the Chi-square test. The p-value < 0.05 was considered significant.

\section{RESULTS}

\section{Macroscopic examination of stool sample}

Table 1 shows $35.98 \%$ stool samples had normal color, $64.01 \%$ had abnormal color, $27.65 \%$ had normal consistency and $72.34 \%$ had abnormal consistency in pregnant women. Blood, mucus and worm were detected in $31.06 \%, 24.62 \%$ and $14.77 \%$ stool samples respectively whereas they were not detected in $68.93 \%, 75.37 \%$ and $85.22 \%$ stool samples of pregnant women respectively.

Table 1: Macroscopic examination of stool sample

\begin{tabular}{|c|c|c|c|c|c|}
\hline \multirow{2}{*}{ Properties } & \multicolumn{4}{|c|}{ Macroscopic examination } & \multirow{2}{*}{ Total } \\
\hline & Normal (\%) & Abnormal (\%) & Presence (\%) & Absence (\%) & \\
\hline Color & $95(35.98)$ & $169(64.01)$ & - & - & \\
\hline Consistency & $73(27.65)$ & $191(72.34)$ & - & - & \\
\hline Blood & - & - & $82(31.06)$ & $182(68.93)$ & 264 \\
\hline Mucus & - & - & $65(24.62)$ & $199(75.37)$ & \\
\hline Worm & - & - & 39 (14.77) & $225(85.22)$ & \\
\hline
\end{tabular}

Prevalence of intestinal parasitosis among pregnant women women was $42 \%$ as shown in figure 1 .

The prevalence of intestinal parasitosis among pregnant

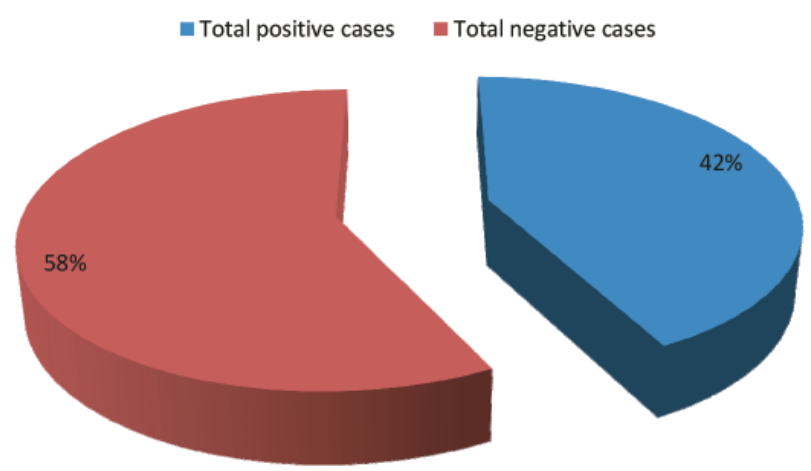

Figure 1. Prevalence of intestinal parasitosis among pregnant women 
Sociodemographic characteristics of pregnant women and its association with intestinal parasites detected and not detected

Table 2 reflects the relationship of age among pregnant women with intestinal parasites detected and not detected which was statistically insignificant ( $p>0.05)$. Similarly, the relationship of religion, residence, occupation and education among pregnant women with intestinal parasites detected and not detected was statistically significant $(\mathrm{p}<0.05)$.

Table 2: Sociodemiographic characteristics of pregnant women and its association with intestinal parasites detected and not detected

\begin{tabular}{|c|c|c|c|c|}
\hline Age (years) & $\begin{array}{l}\text { Intestinal parasites } \\
\text { detected }(n=112)(\%)\end{array}$ & $\begin{array}{l}\text { Intestinal parasites not } \\
\text { detected }(n=152)(\%)\end{array}$ & Total $(\mathrm{N}=264)$ & Statistics \\
\hline $15-19$ & $23(20.53)$ & $18(11.84)$ & $41(15.53)$ & \multirow{5}{*}{$\begin{array}{c}x 2=5.88 p= \\
0.20\end{array}$} \\
\hline $20-24$ & $32(28.57)$ & $36(23.68)$ & $68(25.75)$ & \\
\hline $25-29$ & $19(16.95)$ & $33(21.71)$ & $52(19.69)$ & \\
\hline $30-34$ & 15 (13.39) & $28(18.42)$ & $43(16.28)$ & \\
\hline$>35$ & $23(20.53)$ & $37(24.34)$ & $60(22.72)$ & \\
\hline \multicolumn{5}{|l|}{ Religion } \\
\hline Hindu & $54(48.21)$ & $143(94.07)$ & 197 (74.62) & \multirow{2}{*}{$\begin{array}{c}x 2=71.62 p= \\
0.00001\end{array}$} \\
\hline Muslim & $58(51.78)$ & $9(5.92)$ & $67(25.37)$ & \\
\hline \multicolumn{5}{|l|}{ Residence } \\
\hline Rural & $97(86.60)$ & $86(56.57)$ & $183(69.31)$ & \multirow{2}{*}{$\begin{array}{c}x 2=27.33 p= \\
0.00001\end{array}$} \\
\hline Urban & $15(13.39)$ & $66(43.42)$ & $81(30.68)$ & \\
\hline \multicolumn{5}{|l|}{ Occupation } \\
\hline Employed & $13(11.60)$ & $59(38.81)$ & $72(27.27)$ & \multirow{2}{*}{$\begin{array}{c}x 2=24.06 p= \\
0.00001\end{array}$} \\
\hline Unemployed & 99 (88.39) & $93(61.18)$ & $192(72.72)$ & \\
\hline \multicolumn{5}{|l|}{ Education } \\
\hline Literate & $52(46.42)$ & $45(29.60)$ & $97(36.60)$ & \multirow{2}{*}{$\begin{aligned} x 2 & =7.85 p \\
& =0.005\end{aligned}$} \\
\hline Illiterate & $60(53.57)$ & $107(70.39)$ & $167(63.25)$ & \\
\hline
\end{tabular}

Symptomwise distribution of positive cases and its of intestinal parasites among pregnant women was association statistically significant $(\mathrm{p}<0.05)$ as shown in table 3 .

The association of positive cases with the symptoms

Table 3: Symptomwise distribution of positive cases and its association

\begin{tabular}{lccc}
\multicolumn{1}{c}{ Parameter } & Total no. (\%) & Positive cases (\%) & Statistics \\
\hline Symptomatic & $189(71.59)$ & $91(81.25)$ & \\
Asymptomatic & $75(28.40)$ & $21(18.75)$ & x 2 $=\mathbf{3 . 8 5} \mathbf{p}=\mathbf{0 . 0 4}$ \\
\hline Total & $\mathbf{2 6 4}$ & $\mathbf{1 1 2}$ & \\
\hline
\end{tabular}

Trimester wise distribution of study population and pregnant women with positive cases was statistically its association with parasites detected difference $(p>0.05)$.

Table 4 shows the association of trimesters among

Table 4: Trimester wise distribution of study population and its association with parasites detected

\begin{tabular}{|c|c|c|c|}
\hline Trimesters & Total no. (\%) & Positive cases (\%) & Statistics \\
\hline $1^{\text {st }}$ & $81(30)$ & $22(19.64)$ & \\
\hline $2^{\text {nd }}$ & $86(32.57)$ & $39(76.78)$ & $x 2=5.16$ \\
\hline $3^{\text {rd }}$ & $97(36.74)$ & $51(45.53)$ & $p=0.75$ \\
\hline Total & 264 & 112 & \\
\hline
\end{tabular}

Obstetrics history of pregnant women and its association with positive cases

The association of gravida and parity of pregnant women with positive cases was found to be statistically insignificant $(\mathrm{p}>0.05)$ as shown in table 5 . 
Table 5: Obstetrics history of pregnant women and its association with positive cases

\begin{tabular}{|c|c|c|c|}
\hline Gravida & Total No. (\%) & Positive cases (\%) & Statistics \\
\hline 1 & $66(25)$ & $26(23.21)$ & \multirow{5}{*}{$x 2=1.01 p=0.79$} \\
\hline 2 & $59(22.26)$ & $21(18.75)$ & \\
\hline 3 & $61(23.10)$ & $28(25)$ & \\
\hline 4 & $78(29.54)$ & 37 (33.03) & \\
\hline Total & 264 & 112 & \\
\hline \multicolumn{4}{|l|}{ Parity } \\
\hline 0 & 19 (7.19) & $5(4.46)$ & \multirow{6}{*}{$x 2=9.05 p=0.05$} \\
\hline 1 & $54(20.54)$ & $12(10.71)$ & \\
\hline 2 & $55(20.83)$ & 35 (31.25) & \\
\hline 3 & $71(26.89)$ & $29(25.89)$ & \\
\hline 4 & $65(24.62)$ & $31(27.67)$ & \\
\hline Total & 264 & 112 & \\
\hline
\end{tabular}

Types of parasites detected from stool sample Figure 2 depicts altogether 8 different types of parasites were detected from stool samples of study population. Among the parasite positive samples, 13\% showed presence of Hook worm, followed by $11 \%$ A. lumbricoides, 2\% E. vermicularis, 20\% E. histolytica, $16 \%$ G. lamblia, 6\% Taenia spp., 7\% H. nana and 4\% cyclospora spp. Likewise, 3\% samples contained both Hookworm and Taenia spp., $4 \%$ had Hookworm and $A$. lumbricoides, 2\% had A. lumbricoides and Taenia spp., 4\% had Hookworm and E. histolytica, 3\% had Hookworm and G. lamblia, 4\% had G. lamblia + E. histolytica and 1\% had T. trichura and Hookworm.

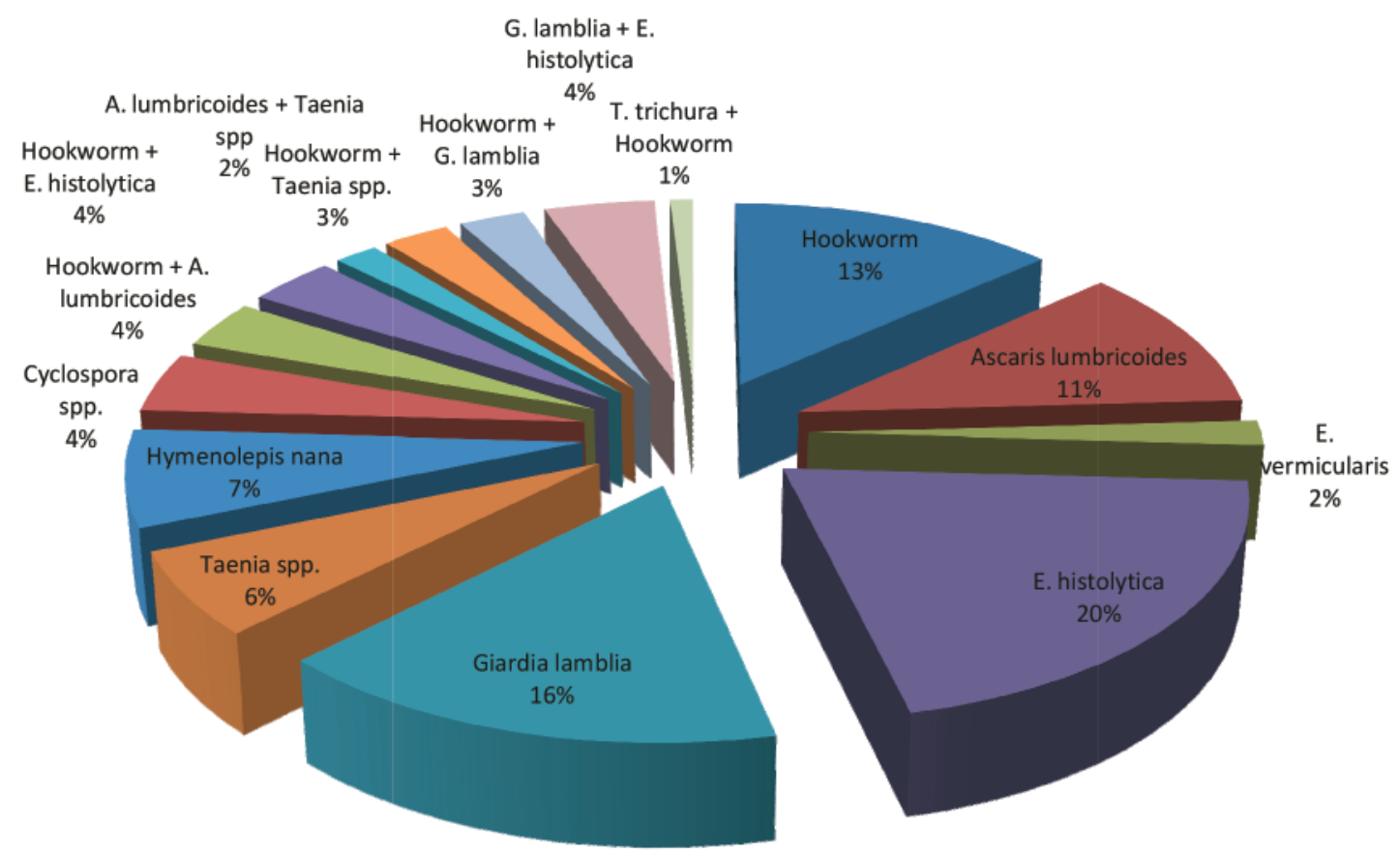

Figure 2: Types of parasites detected from stool sample

\section{Pattern of infection}

Figure 3 shows two different types of intestinal parasitic infection among study population. $80 \%$ single types of parasites were detected and 20\% multiple types of parasites were detected which can cause single and multiple infection. 


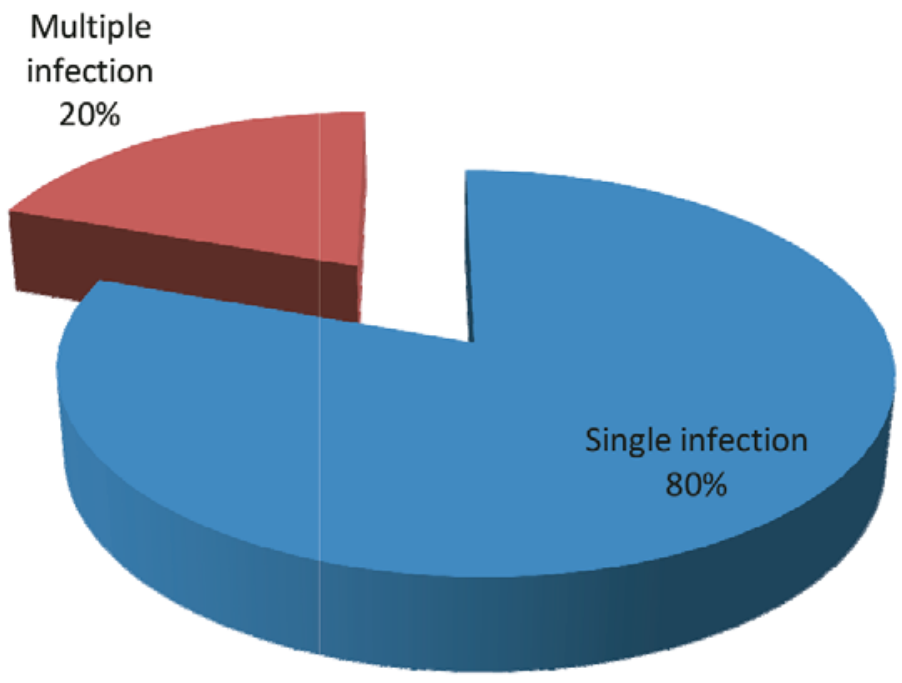

Figure 3: Pattern of infection

Association of trimesterwise study population with single and multiple parasitic infections

The association of trimesters of pregnant women with single and multiple infections was found to be statistically insignificant $(\mathrm{p}>0.05)$ as shown in table 6 .

Table 6: Association of trimesterwise study population with single and multiple parasitic infections

\begin{tabular}{lcccc}
\hline \multicolumn{1}{c}{ Trimesters } & Single infection (\%) & Multiple infections (\%) & Total no. (\%) & p-value \\
\hline $1^{\text {st }}$ & $15(16.66)$ & $7(31.81)$ & $22(19.64)$ & \\
$2^{\text {nd }}$ & $30(33.33)$ & $9(40.90)$ & $39(34.82)$ & $\mathbf{p}=\mathbf{0 . 1 1}$ \\
$3^{\text {rd }}$ & $45(50)$ & $6(27.27)$ & $51(45.53)$ & \\
Total & $\mathbf{9 0}$ & $\mathbf{2 2}$ & 112 & \\
\hline
\end{tabular}

Association of agewise study population with single and multiple parasitic infections

Table 7 reveals the association of age of pregnant women with single and multiple infections which was found to be statistically insignificant $(p>0.05)$.

Table 7: Association of agewise study population with single and multiple parasitic infections

\begin{tabular}{lcccc}
\hline \multicolumn{1}{c}{ Age (years) } & Single infection (\%) & Multiple infections (\%) & Total no. (\%) & p- value \\
\hline $15-19$ & $18(20)$ & $5(22.72)$ & $23(20.53)$ & \\
$20-24$ & $29(32.22)$ & $3(13.63)$ & $32(28.57)$ & \\
$25-29$ & $15(16.66)$ & $4(18.18)$ & $19(16.96)$ & $\mathbf{p}=\mathbf{0 . 5 1}$ \\
$30-34$ & $13(14.44)$ & $2(9.09)$ & $15(13.39)$ & \\
$>35$ & $15(16.66)$ & $8(36.36)$ & $23(20.53)$ & \\
Total & $\mathbf{9 0}$ & $\mathbf{2 2}$ & $\mathbf{1 1 2}$ & \\
\hline
\end{tabular}

Correlation of variables with intestinal parasites in pregnant women

The correlation between all the variables with intestinal parasites detected and not detected was found to be statistically significant $(\mathrm{p}<0.05)$ except consumption of green leafy vegetables was found statistically difference $(\mathrm{p}>0.05)$ as shown in table 8 . 
Table 8: Correlation of variables with intestinal parasites in pregnant women

\begin{tabular}{|c|c|c|c|c|}
\hline Variables & $\begin{array}{c}\text { Intestinal parasites } \\
\text { detected }(n=112)(\%)\end{array}$ & $\begin{array}{l}\text { Intestinal parasites not } \\
\text { detected }(n=152)(\%)\end{array}$ & Total $(\mathrm{N}=\mathbf{2 6 4})$ & p-value \\
\hline \multicolumn{5}{|c|}{ Hand washing before eating with soap and water } \\
\hline Never & $79(70.53)$ & $53(34.86)$ & $132(50)$ & \multirow{3}{*}{$p<0.001$} \\
\hline Sometimes & $23(20.53)$ & $47(30.92)$ & $70(26.51)$ & \\
\hline Most of the times & $10(8.92)$ & $52(34.21)$ & $62(23.48)$ & \\
\hline \multicolumn{5}{|c|}{ Hand washing after defecation } \\
\hline Never & $51(45.53)$ & $12(7.89)$ & $63(23.86)$ & \multirow{3}{*}{$p<0.001$} \\
\hline Sometimes & $43(38.39)$ & $136(89.47)$ & $179(67.80)$ & \\
\hline Most of the times & $18(16.07)$ & $4(2.63)$ & $22(8.33)$ & \\
\hline \multicolumn{5}{|c|}{ Consumption of green leafy vegetables } \\
\hline Never & $6(5.35)$ & $13(8.55)$ & $19(7.19)$ & \multirow{3}{*}{$p=0.15$} \\
\hline Sometimes & $20(17.85)$ & $39(25.65)$ & $59(22.34)$ & \\
\hline Most of the times & $86(76.78)$ & $100(65.78)$ & $186(70.45)$ & \\
\hline \multicolumn{5}{|l|}{ Use of dirty latrines } \\
\hline Never & $25(22.31)$ & $12(7.89)$ & $37(14.01)$ & \multirow{3}{*}{$p<0.001$} \\
\hline Sometimes & 39 (34.82) & $33(21.71)$ & $72(27.27)$ & \\
\hline Most of the times & $48(42.85)$ & $107(70.39)$ & $155(58.71)$ & \\
\hline \multicolumn{5}{|c|}{ Use of footwear outside home } \\
\hline Never & 47 (41.96) & $9(5.92)$ & $56(21.21)$ & \multirow{3}{*}{$p<0.001$} \\
\hline Sometimes & $33(29.46)$ & $4(2.63)$ & $37(14.01)$ & \\
\hline Most of the times & $32(28.51)$ & $139(91.44)$ & $171(64.72)$ & \\
\hline
\end{tabular}

\section{DISCUSSION}

Intestinal parasitosis is one of the most prevalent infectious diseases in women of reproductive age and children in developing countries including Nepal (Nipurte et al. 2020). According to WHO, IPI is considered as a public health problem if its prevalence is greater than $20 \%$ (WHO, 2017). The prevalence of intestinal parasitosis among pregnant women in the present study was 42\%. Previously, Yesuf et al. in 2019, Hailu et al. in 2020, Nipurte et al. in 2020 have reported $43.8 \%, 37.3 \%$ and $42.67 \%$ prevalence. These all findings are almost analogous to the present study. This could be due to the presence of intestinal parasites which is indicative of fecal pollution of soil and domestic water supply due to poor sanitation and improper sewage disposal. Also, it can be attributed to unhygienic practices and lack of awareness of transmission of these intestinal parasites (Nipurte et al. 2020).

In contrast, previous findings reported from Bogota, Colombia (1.2\%) (Espinosa et al. 2018), Nepal (35\%) (Sapkota and Maharjan, 2018), kwale district of Kenya (25.23\%) (Hopkins et al. 2013), Bahirdar, North West Ethiopia (31.5\%) (Derso et al. 2016), Gandhi memorial hospital (25.2\%) (Gebre, 2012) and Debre Markos, North West Ethiopia (27.4\%) (Kumera et al. 2018) have revealed lower prevalence than present study.
These variations could be attributable to smaller sample size, the differences in - socio-demographic status, geographical area and cultural practices, implementation of various intervention strategies, study settings, time of the study, and the methods employed for stool examination. The another possible reason might be due to inappropriate handwashing practice, poor shoe wearing habit and difference in existing sanitation facilities and practices.

The result of this study depicts maximum number of parasites detected in age between $20-24$ years $(28.57 \%)$ followed by age group greater than 35 years (20.53\%) and $25-29$ years (16.95\%). Nipurte et al. in 2020 observed the parasites were seen predominantly in the age group $34-40$ years (50\%) followed by $26-33$ years $(44.7 \%)$ and $18-25$ years $(40.4 \%)$. Studies conducted by Alli et al. in 2011 and Usip et al. in 2017 also showed similar findings which is not in accordance with the present study. This might be due to the variation in study population's age group size, food habit behaviour and hygiene practice. The association of intestinal parasites detected in relation to age was found to be statistiscally difference $(p=0.20)$.

The present study reports the higher incidence of intestinal parasites in pregnant women was found in Muslim compared to Hindu religion. Maximum 
participants were infected from the rural area and those who were unemployed. Also, other related studies in pregnant women carried out by Derso et al. in 2016 and Mahande et al. in 2016 had accounted predominance in rural populations which is in accord with the present study. The prospects might be due to low socioeconomic status, the surrounding environmental factors, level of sanitation and hygiene would have been similar to that of a rural set up which are the major confounding factors for the transmission of intestinal parasitic infection. But, Nipurte et al. in 2020 observed the prevalence of intestinal parasites was almost similar in both rural and urban women contrast to our study (Nipurte et al. 2020).

Education is considered as one of the most basic strategies for health improvement and promotion of quality of life (Mirzaee et al. 2013). The present study showed mostly illiterate participants were infected. Similar findings were also reported by Obiakor-Okeke et al. in 2014 and Hailu et al. in 2020. This might be due to the lack of knowledge, low educational level, unawareness about infections and non-hygienic practices of pregnant mothers which ultimately increase risk of infections. The association of intestinal parasites detected in relation to religion, residence, occupation and education was statistiscally significant $(\mathrm{p}<0.05)$.

This study reveals the highest number (71.59\%) of intestinal parasites detected in those who have symptoms were statistically significant $(p=0.04)$. But, the study conducted in Venezuela by Morales et al. in 2006 observed a high prevalence of intestinal parasitosis (more than $70 \%$ ) those who had no symptoms which are not in harmony with the present study. This might be due to the transversal analysis of pregnant women attending to prenatal control outpatient health care centers in Venezuela which have the larger study population (Morales et al. 2006).

In this study, maximum study participants were infected in $3^{\text {rd }}$ trimester of pregnancy followed by $2^{\text {nd }}$ trimester of pregnancy which was statistically insignificant $(p=0.75)$. Since, it is expected to have lower level of haemoglobin in $3^{\text {rd }}$ trimester which is a physiological process in pregnancy. There is no evidence that the cause of infection in $3^{\text {rd }}$ trimester of pregnancy established as to whether it is due to worm infestation or due to physiological cause with superimposed worm infestation (Raut et al. 2016).

The mothers of younger children are expected to come in close contact with their children during their daily activities leading to the more prevalence of infection. The findings of this study depict the maximum number of participants infected with parasites who had four gravida followed by the three, one and two. Higher numbers of respondents were infected those who were multiparous. Similar findngs were also attained in previous studies conducted by Nipurte et al. in 2020 and Alli et al. in 2011. The infection becomes more severe in women who are pregnant for the first time (primigravida) compared with other gravida as reported by Muhangi et al. in 2007. The association of gravida and parity of pregnant women with positive cases was found to be statistically insignificant $(p=0.79$ and $\mathrm{p}=0.05$ ) respectively.

Women may even acquire parasitic infections in the process of growing the family's food where insufficiently composted human faeces may be used as fertilizer on vegetable crops (Humphries et al. 1997). In the present study, altogether 8 different types of parasites were detected from stool samples. The most predominant parasites noted during pregnancy were E. histolytica (20\%), followed by G. lamblia (16\%) and chased by Hook worm (13\%) and A. lumbricoides with $11 \%$. Infection with $E$. histolytica is common inhabitants of developing countries and predominantly affects people with poor socio-economic conditions, nonhygienic practices and malnutrition (Braga et al. 2001).

Infections with E. histolytica, G. lamblia, hookworm, and A. lumbricoids parasites are the most common infection in rural areas, and their transmission is closely associated with socio-economic status, poor sanitation, and absence of adequate safe drinking water supplies (Merid et al. 2001). G. lamblia was found to be the second most common protozoan among study participants in current study. The highest prevalence of Giardia indicates the poor sanitary and personal hygienic condition of the respondents. Furthermore, the cyst of G. lamblia is resistant to the normal level of chlorination, and therefore, it can be easily transmitted through drinking water. The common causes of acute or persisting diarrhoea in people which interferes with intestinal absorption nutrients and growth rate of children (Yadav and Prakash, 2016).

The present study reflects the highest number of 
protozoa were detected whereas less number for helminths. A similar study conducted by Hailu et al. in 2020 in West Gojjam Zone, Northwest Ethiopia also observed the prevalence of intestinal protozoa was higher than helminths which are coexisting with the present study. The least number of helminths were detected which might be due to the differences in the distribution of helminths from place to place or from one geographical area to another. The temperature, soil type, rainfall, altitude, and humidity are also the major environmental factors that influence the pre-existence of helminthic infections in one geographical area (De Silva, 2003).

But, a study done in Gondar town, Northwest Ethiopia the presence of protozoa detected was fewer (Alem et al. 2013) which is distinct with this study. The differences might be due to the disparity in the detection method used to identify intestinal parasites. FECT which has higher sensitivity than direct microscopy was used as means of diagnosis in the present study.

The current study depicts Hookworm infection among pregnant women was $13 \%$. Hailu et al. in 2020 reported $18.6 \%$ hookworm attacked to pregnant women slightly higher not in accord with this study. The difference might be due to the diversity in shoewearing habit and the level exposed to contaminated soil with hookworm larvae that penetrate the human skin. Working bare hands and walking barefoot are the major means of transmission for hookworm infection. But, a similar study conducted by Roberts et al. 2011 in Nepal reported the same trend parallel to our study (Roberts et al. 2011).

The present study depicts $11 \%$ A. lumbricoids infected the study participants. Nalini et al. 2017 reported the most prevalent ascariasis infestation was $76.5 \%$ during pregnancy period incongruent with this study which might be due to larger study population size. Larocque et al. 2005 also accounted ascariasis as the most common infestation in pregnant women. A. lumbricoids may cause intestinal obstruction, liver abscess, local irritation, and damage with malabsorption as main cellular related events associated with the infection (Fuseini et al. 2009). A. lumbricoides plays an important role in precipitating protein-energy malnutrition in undernourished children (Fuseini et al. 2009).

The other explanations are that the Ascaris ova are also spread by coprophilous animals and can be carried to areas away from defecation sites (Obiamiwe et al. 1991) their eggs resist drying and can survive for long periods in soil. Being coated with mucopolysaccharides ascaris eggs powerfully adheres to different surfaces (Awolaju et al. 2009).

This study also depicted attention to lower prevalence of H. nana and Taenia spp. which is worldwide parasitic disease with great importance. Lower prevalence was observed in this study which may be due to difficulty in identification of larva. Also, it might be due to rarely transmission occurred from the ingestion of food contaminated with fleas harbouring the cysticercoid larvae. The occurrence of Taenia may be due to risk factors associated with eating raw or insufficiently cooked pork, raw vegetables grown in field fertilized with human faeces contaminated with eggs of T. solium (Yadav and Prakash, 2016).

This study shows $80 \%$ single types of parasites and $20 \%$ multiple types of parasites were detected which can cause single and multiple infection. Similar findings were also obtained in the study conducted by Hailu et al. in 2020 .

This study reveals maximum number of study participants was infected from single infection in $3^{\text {rd }}$ trimester of pregnancy whereas multiple infections were found in $2^{\text {nd }}$ trimester of pregnancy. The association of trimesters of pregnant women with single and multiple infections was statistically insignificant ( $p=0.11$ ). This might be due to the fact that parasitic infection could occur at any stage of the three trimesters during pregnancy, but infection during the first trimester is associated with more severe fetal and placental consequences than those occurring later in pregnancy (Muhangi et al. 2007).

The present study established more number of single infections was in age between 20-24 years followed by 15-19 years whereas multiple infections was detected in greater than 35 years. The association of age of pregnant women with single and multiple infections was observed statistically insignificant $(p=0.51)$. The probable reason is that the health status of young women who are underweight or stunted, those with anemia through its multiple causes or chronic infection will start a pregnancy at great disadvantage of intestinal helminth infection, iron deficiency, and malaria are at increased risk of delivering low birth weight infants. (Steketee et al. 2001) 
Hands are the main pathways of germ transmission. The present study shows the intestinal parasites detected in pregnant women who never washed their hands with soap and water before eating was $70.53 \%$. But, women washing their hands most of the times with soap and water before eating were only $8.92 \%$. The correlation between hand washing before eating with soap and water with intestinal parasites detected and not detected was found to be statistically significant $(p=0.00001)$. The result of this study is almost in accord with the study carried by Nipurte et al. 2020 . This prospect may be due to the fact that washing hands before eating a meal is a simple and effective method of infection prevention and protection against germs and illness. In contrast, Raut et al. 2016 found $32.7 \%$ respondents had the habit of handwashing with soap and water frequently (Raut et al. 2016) which is distinctive with the present study.

This study showed the pregnant women who washed their hands regularly after defecation had lesser $(16.07 \%)$ probability of intestinal parasites followed by women $(38.39 \%)$ who sometimes washed hands and the probability was highest in women (45.53\%) who never washed hands after defecation. The reason may be that critical hand washing is preferred as the best washing practices. Other possibility may be due to traditional practice and understanding the importance of cleaning and washing hands with after defecation. The correlation between hand washing after defecation with intestinal parasites detected and not detected was found to be statistically significant $(p=0.00001)$. These findings are similar to the study conducted by Nipurte et al. (2020), Derso et al. (2016) and Mengist et al. (2017).

Pregnancy requires extra nutrients, especially iron, and produces a "physiological anemia" due to hemodilution (Derso et al. 2016). In the present study, the women with high intake of green leafy vegetable were more prone to intestinal parasitic infections which are in accord with the findings of Nipurte et al. in 2020 and Hailu et al. in 2020. Similar findings were also reported in Northwest Ethiopia (Derso et al. 2016) and East Wolega, Ethiopia (Shiferaw et al. 2015). The correlation between consumption of green leafy vegetables with intestinal parasites detected and not detected was statistically insignificant ( $p=0.15)$. This might be due to the lack of awareness and the absence of education. Also, it may be due to the pregnant women involved in agriculture and cultivation has limited knowledge about how and when intestinal parasites are transmitted. As a result, eating raw vegetables, open defecation, living in unclean environment, and food with soil during pregnancy are a common phenomenon of infection.

But, in a study conducted by Dutta et al. in 2013 showed that dietary practice of taking green leafy vegetables and fruits had protective effect during pregnancy which is not similar to this study. This depicts that the pregnant women should be advised to have plenty of green leafy vegetables but emphasis should be made on washing it thoroughly before consumption.

In the present study, the occurrence of parasitic infection in pregnant women who used dirty latrine repeatedly was more and reduces the occurrence of infection in women who never used dirty latrines and women who sometimes used dirty latrines. The correlation between use of dirty latrines with intestinal parasites detected and not detected was found to be statistically significant $(\mathrm{p}=0.00001)$. These findings are similar to the study conducted by Nipurte et al. 2020 . This may be due to lack of cleanliness and poor hygiene practices.

Regarding to the mode of infection of hookworm, the present study evaluated the prevalence of hookworm infection was more in women who never wore footwares $(41.96 \%)$ followed by those who wore footwares sometimes $(29.46 \%)$ as compared to those who wore most of the time (28.51\%). This might be due to avoiding the personal hygiene like barefoot walking which helps in provoking the infections with soil transmitted helminths like hookworm. In similar type of study, Nipurte et al. in 2020 reported the prevalence of hookworm infection was more in women who never used sandals $(7.5 \%)$ as compared to those who used sandals frequently $(1 \%)$. The correlation between use of footwears with intestinal parasites detected and not detected was found to be statistically significant $(p=0.00001)$.

Tesfaye et al. in 2015 and Lorocque et al. in 2005 have also noted positive correlation between barefoot walking and prevalence of hookworm infection which is similar to the present study. This might be due to manipulating the irrigation activity, barefoot and bare hands which leads to parasites like hookworm to enter by skin penetration. Moreover, water for irrigation is not clean and individuals who have the habit of eating food after cleaning their hands with such water have a 
possibility to ingest the parasites.

\section{CONCLUSION}

This study concluded that the low hygiene and sanitation habits with lack of awareness about intestinal parasitic infections were the major determinant factors for the moderate prevalence of protozoan infection during pregnancy. E. histolytica followed by G. lamblia were predominant parasites. The highest number of single type of parasites was identified.

There was positive association of cases with the symptoms of intestinal parasites but negatively associated with trimesters. The relationship of age and trimesters of pregnant women with single and multiple infections was statistically insignificant. Further, the correlation between all the variables with intestinal parasites detected and not detected was statistically significant except for age and consumption of green leafy vegetables. Strengthening the existing water, sanitation and hygiene programs and public health measures like routine deworming of pregnant mothers should be encouraged in community setting to minimize the burden of intestinal parasitic infection.

\section{LIMITATIONS}

This study limits with the small sample size and uses only single stool specimen to assess infection status avoiding the assessment of the HIV-status and anemia among study participants. Further, more research is recommended to conduct with a large sample size in this region among pregnant women.

\section{ACKNOWLEDGEMENTS}

Our special thanks go to all the pregnant women who participated in this study. We also express our heartfelt gratitude to Department of Obstetrics and Gynecology, Department of Clinical Pathology and Laboratory Medicine, and Hospital management of Ram Janaki Hospital, Janakpurdham for all the logistic supports during this research.

\section{CONFLICT OF INTERESTS}

The authors declare that there is no conflict of interests regarding the publication of this paper.

\section{REFERENCES}

Alem M, Enawgaw B, Gelaw A, Kenaw T, Seid M, and Olkeba Y (2013) Prevalence of anemia and associated risk factors among pregnant women attending antenatal care in Azezo Health Center Gondar Town, Northwest Ethiopia. Journal of
Interdisciplinary Histopathology 1(3):137-144.

Alli JA, Okonko IO, Kolade AF, Nwanze JC, Dada VK, Ogundele M (2011) Prevalence of intestinal nematode infection among pregnant women attending antenatal clinic at the University College Hospital, Ibadan, Nigeria. Advan Appl Sci Res 2(4):1-3.

Alli JA, Okonko IO, Kolade AF, Nwanze JC, Dada VK, Ogundele M (2011) Prevalence of intestinal nematode infection among pregnant women attending antenatal clinic at the University College Hospital, Ibadan, Nigeria. Advan Appl Sci Res 2(4):1-3.

Awolaju BA, Morenikeji OA (2009) Prevalence and intensity of intestinal parasites in five communities in south-west Nigeria. African Journal of Biotechnology 8(18):4542-4546.

Braga LL, Gomes ML, Da Silva MW, Façanha FE, Fiuza L and Mann BJ (2001) Household epidemiology of Entamoeba histolytica infection in an urban community in northeastern Brazil. Am Trop Med Hyg J 65:268-271.

De Silva NR, Brooker S, Hotez PJ, Montresor A, Engels D, Savioli L (2003) Soil-transmitted helminth infections: updating the global picture. Trends in parasitology 19(12):547-51.

Derso A, Nibret E, Munshea A (2016) Prevalence of intestinal parasitic infections and associated risk factors among pregnant women attending antenatal care center at Felege Hiwot referral hospital, Northwest Ethiopia. BMC Infect Dis 16(1):530.

Dotters-Katz S, Kuller J, Heine RP (2011) Parasitic infections in pregnancy. Obstetrical and Gynecol Survey 66(8):515-25.

Dutta S, Chatterjee S, Sinha D, Pal B, Basu M, Dasgupta A (2013) Correlates of anaemia and worm infestation among rural pregnant women: a cross sectional study from Bengal. Literacy 3(11):10-89.

Espinosa Aranzales AF, Radon K, Froeschl G, Pinzón Rondón ÁM, Delius M (2018) Prevalence and risk factors for intestinal parasitic infections in pregnant women residing in three districts of Bogotá, Colombia. BMC Public Health 18(1):1071.

Finkelman FD, Urban JF (2001). The other side of the 
coin: The protective role of the TH2 cytokines. Journal of Allergy and Clinical Immunology 107(5):772-780.

Fuseini G, Edoh D, Kalifa BG, and Knight D (2009) Plasmodium and intestinal helminths distribution among pregnant women in the Kassena-Nankana District of Northern Ghana. Journal of Entomology and Nematology 1(2):019-024.

Gebre W (2012) Intestinal parasitic infection in pregnant women attending antenatal care at Gandhi Memorial Hospital, Addis Ababa, Ethiopia.

Hailu T, Abera B, Mulu W, Kassa S, Genanew A, and Amor A (2020) Prevalence and Factors Associated with Intestinal Parasitic Infections among Pregnant Women in West Gojjam Zone, Northwest Ethiopia. Journal of Parasitology Research: 1-6

Humphries DL, Stephenson LS, Pearce EJ, The PH, Dan HT, Khanh LT (1997) The use of human faeces for fertilizer is associated with increased intensity of hookworminfection in Vietnamese women. Transactions of the Royal Society of Tropical Medicine and Hygiene 91(5):518-520.

Khor LG. Update on the prevalence of malnutrition among children in Asia (2003) Nepal Medical College Journal 5(2)113-122.

Raut KB, Silwal K, Pun KM (2016) Intestinal Worm Infestation and Anaemia in Pregnant Women. $J$ Nepal Med Assoc 54(201):29-32.

Kumera G, Haile K, Abebe N, Marie T, Eshete T (2018) Anemia and hookworm infection among pregnant women attending antenatal care at Debre Markos Referral Hospital, Northwest Ethiopia. PloS One 13(11):1-14.

Larocque R, Casapia M, Gotuzzo E, Gyorkos TW (2005) Relationship between intensity of soiltransmitted helminth infections and anemia during pregnancy. Am J Trop Med Hyg 73: 783-89.

Mahande AM, Mahande MJ (2016) Prevalence of parasitic infections and associations with pregnancy complications and outcomes in northern Tanzania: a registry-based crosssectional study. BMC Inf Dis 16(1):78.

Mehraj V (2006) Prevalence of and factors associated with intestinal parasites among children from
1 to 5 years of age in an urban slum of Karachi, (Ph.D Thesis) Age Khan University, Department of Community Health Sciences.

Mengist HM, Zewdie O, Belew A (2017) Intestinal helminthic infection and anemia among pregnant women attending ante-natal care (ANC) in East Wollega, Oromia, Ethiopia. BMC Res Notes 10(1):440.

Merid Y, Hegazy M, Kekete G, and Teklemariam S (2001) Intestinal helminthic infection among children at Lake Awassa Area, South Ethiopia. The Ethiopian Journal of Health Development (EJHD) 15:31-38.

Mirzaee K, Taghi Shakeri M (2015) Maternal knowledge on postpartum care in healthcare centers of Mashhad, Iran in 2013. J Midwifery Reprod Health 3(4): 456-64

Muhangi L, Woodburn P, Omara M, Omoding N, Kizito D, Mpairwe H, Nabulime J, Ameke C, Morison LA, Elliott AM (2007) Associations between mild to moderate anaemia in pregnancy and helminth, malaria and HIV infection in Entebbe, Uganda. Trans R Soc Trop Med Hyg 101:899-907.

Nalini S, Das R, Singh SA (2017) A Study of Helminthiasis in Pregnancy Sch. Acad J Biosci 5(11):800-803.

Nipurte R, Paranjpe S, Koticha A, Nataraj G, Mehta P (2020) Correlation of sociodemographic factors and intestinal parasites in pregnant women. Int J Res Med Sci 8:244-51.

Obiakor-Okeke, Nwaogu NP, Tina C, Agugo UA, Odigwe Nkechi M (2014) The prevalence of intestinal parasites and anaemia among pregnant women attending antenatal clinic in Federal Medical Centre Owerri, Imo State, Nigeria. J Biol, Agriculture Health 4(25):171-7.

Obiamiwe BA, Nmorsi P (1991) Human gastrointestinal parasites in Bendel State, Nigeria. Angewandte Parasitologie 32(3):177-83.

Ohaeri CC and Orji BN (2013) Intestinal parasites among undergraduate students of Michael Okpara University of Agriculture, UmudikeAbia state, Nigeria. World Applied Sciences Journal 25(8):1171-1173.

Rai SK, Hirai K, Abe A (2002) Intestinal parasitosis 
among school children in a rural hilly area of the Dhading district, Nepal. Nepal Med Coll J 4:54-58.

Roberts TK, Gravett CA, Velu PP et al. (2011) Epidemiology and aetiology of maternal parasitic infections in low- and middle-income countries. Journal of Global Health 1(2): 189-200.

Rodr'iguez-Morales A, Barbella AR, Case C, Arria M, Ravelo M, Perez H, Oscar Urdaneta O, Gervasio $G$ et al. (2006) Intestinal Parasitic Infections among PregnantWomen in Venezuela Hindawi Publishing Corporation Infectious Diseases in Obstetrics and Gynecology Article ID 23125:1-5.

Sapkota L, Maharjan M (2018) Anemia association with intestinal parasitic infection in pregnant women attending antenatal clinic at Tribhuvan University Teaching Hospital. JACEM 3:41-7.

Shiferaw BM and Mengistu DA (2015) Helminthiasis: hookworm infection remains a public health problem in Dera District, South Gondar, Ethiopia, PLoS ONE 10(12) article e0144588.

Steketee RW, Nahlen BL, Parise ME \& Menendez C (2001) The burden of malaria in pregnancy in malaria-endemic areas. Am J Trop Med Hyg 64(Suppl. 1-2):28-35.

Steketee WR (2003) Pregnancy, nutrition and parasitic diseases. The Journal of Nutrition 133(5):1661S-1667S.

Stephenson SL, Latham CM, and Ottesen AE (2000) Malnutrition and parasitic helminth infections. Parasitology 121(S1): S23-S38.
Tesfaye DJ, Beshir WG, Dejene T, Tewelde T (2015) Prevalence of intestinal helminthiases and associated factors among pregnant women attending antenatal clinic of Nigist Eleni Mohammed memorial hospital, Hossana, Southern Ethiopia. Open Access Library J 31;2(7):11.

Usip, Esiet LP, Udeme UA, Nseabasi AM (2017) Prevalence of intestinal parasites among pregnant women attending antenatal clinic in General Hospital Calabar, Nigeria. Am J Res Com 5(7):46.

WHO, "Soil transmitted helminths," Fact sheet, 2014, September 2017.

World Health Organization. Intestinal worms (2017).

Yadav K and Prakash S (2016) Study on Intestinal Parasitic Infections in Muslim community of Janakpurdham, Nepal. Janaki Medical College Journal of Medical Sciences 4(1): 36-45.

Yadav K, Prakash S (2016) Study of Intestinal Parasitosis among School Children of Kathmandu Valley, Nepal. Asian Journal of Biomedical and Pharmaceutical Sci 6(59):40-47.

Yakasai AI, Umar UA (2013) A review parasitic infestation in pregnancy. Asian J Natural Appl Sci 2(1):31-8.

Yesuf AD, Abdissa TL, Gerbi AE and Tola KE (2019) Prevalence of intestinal parasitic infection and associated factors among pregnant women attending antenatal care at public health facilities in Lalo Kile district, Oromia, Western Ethiopia BMC Res Notes 12:735. 